\title{
Cerebellar Gray Matter Volume Correlates with Duration of Cocaine Use in Cocaine-Dependent Subjects
}

\author{
Minyoung E Sim', In Kyoon Lyoo*, 1,2, Chris C Streeter ${ }^{3}$, Julie Covell' ${ }^{2}$ Ofra Sarid-Segal ${ }^{3}$, Domenic A Ciraulo ${ }^{3}$, \\ Minue J Kim ${ }^{1,2}$, Marc J Kaufman ${ }^{2}$, Deborah A Yurgelun-Todd ${ }^{2}$ and Perry F Renshaw ${ }^{2}$ \\ 'Department of Psychiatry, Seoul National University College of Medicine, Seoul, South Korea; ${ }^{2}$ Department of Psychiatry, McLean Hospital Brain \\ Imaging Center, Harvard Medical School, Belmont, MA, USA; ${ }^{3}$ VA Boston Healthcare System, Department of Psychiatry, Boston University School \\ of Medicine, Boston, MA, USA
}

\begin{abstract}
This study was conducted to explore differences in gray and white matter volume between cocaine-dependent and healthy comparison subjects using optimized voxel-based morphometry (VBM). Brain magnetic resonance imaging (MRI) and neuropsychological function tests were performed for 40 cocaine-dependent subjects ( $41.4 \pm 6.9$ years, 27 men) and 41 healthy age- and sex-matched comparison subjects $(38.7 \pm 8.8$ years, 26 men). Optimally normalized whole brain MR images were segmented, modulated, smoothed, and compared between groups with statistical parametric mapping. The cocaine-dependent group had lower gray matter volumes in bilateral premotor cortex (Brodmann area (BA) 6, 8; 16.6\%), right orbitofrontal cortex (BA 10, I5.1\%), bilateral temporal cortex (BA 20, 38; 15.9\%), left thalamus (12.6\%), and bilateral cerebellum (13.4\%) as well as lower right cerebellar white matter volume (I0.0\%) relative to the comparison group at a corrected $p<0.05$ for multiple comparisons. Duration of cocaine use negatively correlated with right and left cerebellar gray matter volumes $(r=-0.37, r=-0.39$, respectively). In cocaine-dependent subjects, lower cerebellar hemispheric gray and white matter volumes were correlated with deficits in executive function and decreased motor performance. This study reports that cocaine-dependent subjects have lower gray matter volumes in cerebellar hemispheres as well as in frontal, temporal cortex, and thalamus. These findings are the first to suggest that the cerebellum may be vulnerable to cocaine-associated brain volume changes, and that cerebellar deficits may contribute to neuropsychological deficits and motor dysfunction frequently observed in cocaine-dependent subjects.

Neuropsychopharmacology (2007) 32, 2229-2237; doi:I 0. I038/sj.npp. I 30I346; published online I4 February 2007
\end{abstract}

Keywords: cocaine; cerebellum; optimized voxel-based morphometry

\section{INTRODUCTION}

The behavioral effects of cocaine have been linked to the modulation of dopamine release in mesocorticolimbic dopaminergic pathways (Kuhar et al, 1991). Consequently, prior brain-imaging studies of cocaine abusers have focused on abnormalities in mesocorticolimbic areas including frontal and temporal cortex, striatum, and limbic regions (Franklin et al, 2002; Lyoo et al, 2004; Matochik et al, 2003).

Recently, several quantitative structural brain imaging studies have been conducted in cocaine abusers including two utilizing the standard voxel-based morphometry (VBM) technique (Franklin et al, 2002; Matochik et al, 2003).

Preliminary results from this study were presented at the College on Problems of Drug Dependence Annual Meeting, held in San Juan, Puerto Rico, June 12-17, 2004.

*Correspondence: Dr IK Lyoo, Department of Psychiatry, Seoul National University College of Medicine, 28 Yongon-dong, Chongnogu, Seoul I I0-744, South Korea, Tel: +82 22072 3173, Fax: +82 2 3672 0677, E-mail: inkylyoo@yahoo.com

Received 13 April 2006; revised 17 November 2006; accepted 15 December 2006
Franklin et al (2002) first reported that cocaine-dependent subjects had gray matter density decreases in frontotemporal brain regions including orbitofrontal, anterior cingulate, insular, and superior temporal cortex relative to comparison subjects. Matochik et al (2003) subsequently reported in a VBM study with regions of interest confined to frontal brain areas that cocaine-dependent subjects had gray matter density decreases in regions including cingulate gyrus and orbitofrontal cortex. In addition, frontal white matter abnormalities have been reported in cocaine abusers (Lim et al, 2002; Lyoo et al, 2004).

Although these findings indicate that the frontal lobes are a major target for cocaine's effects, prior functional brain-imaging studies in cocaine-dependent subjects noted impairments in other brain areas including the cerebellum (Gottschalk and Kosten, 2002) as well as in prefrontal and limbic cortex (Ernst et al, 2000; Volkow et al, 1988). An animal study has also reported cocaine-induced microscopic lesions in cerebellum (Barroso-Moguel et al, 2002).

Cerebellar dysfunction may result in neuropsychological deficits as well as impairments in fine motor control that have been observed in cocaine abusers (Gottwald et al, 2004; 
Hester and Garavan, 2004; Smelson et al, 1999). Yet, to date, there have been no imaging studies suggesting the presence of cerebellar structural abnormalities in cocaine-dependent humans.

Accordingly, we conducted a VBM study in a large cohort of cocaine-dependent subjects (40 cocaine-dependent and 41 comparison subjects). We utilized an optimized VBM technique (Good et al, 2001b), which incorporates additional spatial processing steps that improve image registration and segmentation, as compared to the standard VBM technique (Ashburner and Friston, 1997). Optimized VBM reduces voxel misclassification errors (Good et al, 2001b) and offers increased sensitivity (Keller et al, 2004) over the standard VBM technique.

Based on previous functional, animal, and magnetic resonance imaging (MRI) studies which have reported decreased cerebellar blood flow in cocaine-dependent subjects (Gottschalk and Kosten, 2002), cocaine-induced cerebellar pathology in animal (Barroso-Moguel et al, 2002) and regional gray matter concentration or density reductions (Franklin et al, 2002; Matochik et al, 2003) in cocaine abusers, the possible differences between the cocainedependent subjects and the healthy comparison subjects including lower gray matter volumes in the cerebellum, frontal, and temporal cortex, are compared in this study.

\section{MATERIALS AND METHODS}

\section{Subjects}

Eligible subjects were cocaine-dependent persons between 18 and 60 years of age enrolled in two National Institute on Drug Abuse (NIDA)-sponsored treatment studies. Cocainedependent subjects met criteria for a DSM-IV diagnosis of cocaine dependence as determined by Structured Clinical Interview for DSM-IV Axis I Disorders, Research Version, Nonpatient Edition (SCID) (First et al, 1997) and reported using cocaine on at least six occasions within the 28-day period before screening. Self-report of current cocaine use was substantiated with at least one urine specimen testing positive for cocaine metabolites over a 2 -week period before study entry. Potential cocaine-dependent subjects were excluded if they had a (1) current dependence on any psychoactive drug other than cocaine, alcohol, or nicotine, (2) neurological or psychiatric disorders requiring immediate treatment, (3) schizophrenia or bipolar illness, (4) serious medical illness, (5) prescribed psychoactive medications, (6) asthma, (7) amenorrhea due to pregnancy, (8) abnormal laboratory results during screening, or (9) contraindications to scanning. Cocaine-dependent subjects with a current history of alcohol dependence not requiring medical detoxification were accepted.

Age- and sex-matched healthy comparison subjects recruited by newspaper advertisement and by word of mouth were screened for Axis I disorders using the SCID. Control subjects were required to have no history of cocaine or alcohol dependence/abuse and no history of Axis I or neurological disorders.

Owing to the high comorbidity rate of cocaine and alcohol dependence in the cocaine-dependent subjects (Miller et al, 1989), the following additional exclusion criteria were applied to determine if cocaine-dependent subjects with histories of alcohol abuse or dependence were eligible for enrollment: subjects with liver function tests greater than three times normal values, subjects with histories of a complicated alcohol withdrawal, subjects requiring medical detoxification or not identifying cocaine as their drug of choice.

This study was approved by the Institutional Review Boards of Boston Medical Center, McLean Hospital, the Boston VA Healthcare System, and Seoul National University Hospital. After complete description of the study to the subjects, written informed consent was obtained.

\section{MR Image Acquisition}

Brain MRI was performed using a 1.5 whole-body imaging system (Horizon Echo-Speed, General Electric Medical Systems, Milwaukee, WI, USA) and a custom-made linear birdcage coil with approximately $40 \%$ improvement in signal-to-noise ratio and improved homogeneity over standard quadrature head coils (Dager et al, 2004). A three-dimensional spoiled-gradient echo pulse sequence was used to produce 124 contiguous $1.5-\mathrm{mm}$-thick coronal images (echo time $(\mathrm{TE})=5 \mathrm{~ms}$, repetition time $(\mathrm{TR})=$ $35 \mathrm{~ms}, 256 \times 192$ matrix; field of view $(\mathrm{FOV})=24 \mathrm{~cm}$, flip angle $=45^{\circ}, 1$ number of excitations (NEX)). Axial protondensity and T-2 weighted images $(\mathrm{TE}=30 / 80 \mathrm{~ms}, \mathrm{TR}=$ $3000 \mathrm{~ms}, 256 \times 192$ matrix; FOV $=24 \mathrm{~cm}$, flip angle $=45^{\circ}$, 0.5 NEX, 3-mm-thick slices, no skip) were obtained.

These images were screened by an experienced neuroradiologist, who was blind to subject grouping, for brain structural abnormalities. Three subjects from the cocaine group and two from the comparison group had qualitative abnormalities including cerebral atrophy, enlarged ventricles, or white matter hyperintensities with a severity greater than grade II by Fazekas' criteria. All were excluded from the study. Consequently, 40 cocaine-dependent subjects and 41 comparison subjects were included in the VBM part of study.

\section{Optimized VBM}

Customized gray and white matter templates were created by averaging all the 81 smoothed normalized gray and white matter images, respectively, to produce optimized normalization parameters (Good et al, 2001b). Original structural MR images were segmented into gray and white matter images, followed by an extraction of unconnected nonbrain voxels of scalp, skull, or venous sinuses from segmented images (Good et al, 2001a). Each segmented gray/ white matter image was normalized to its own gray and white matter templates, respectively. To facilitate optimal segmentation, optimized normalization parameters were reapplied to the original whole-brain structural images. The spatial normalization process consists of 12 parameter affine transformations (Good et al, 2001a), followed by nonlinear warping using basis functions $(7 \times 8 \times 7)$. Optimally normalized whole brain images were then segmented and extracted repeatedly to eliminate any remaining nonbrain voxels on segmented gray and white matter images.

To preserve the potential volume changes developed in the spatial normalization process, segmented images were 
modulated by the Jacobian determinants obtained from the spatial normalization procedure (Good et al, 2001a). This step enables the measurement of the absolute volumes as compared with the relative concentration or density in the standard VBM technique. Each optimally normalized, segmented, modulated gray/white image was smoothed with an isotropic Gaussian kernel with $8 \mathrm{~mm}$ full width-half maximum (FWHM). Significant differences were estimated using random Gaussian field theory (Worsley et al, 1996). Parameters to define regions of significant differences included $p$-value $<0.05$ (corrected for multiple comparisons) and the extent threshold $=400$ isotropic voxels of $1.0 \mathrm{~mm}^{3}$ (equivalent of 50 isotropic voxels of $2.0 \mathrm{~mm}^{3}$ ).

The optimized VBM method is rater-independent as the predefined procedure set is sequentially conducted. In addition, the rater was blind to clinical information regarding study subjects. Statistical parametric mapping (SPM2) (Wellcome Department of Imaging Neuroscience, London, UK) was executed in MATLAB 7.1 (MathWorks, Natick, MA, USA).

\section{Neuropsychological Function Tests}

A battery of neuropsychological function tests was conducted by a trained neuropsychologist. The following tests were performed: Trails A, a simple test of psychomotor speed; and Trails B, a more difficult test of executive function (Reitan, 1992). To examine further executive function, the Stroop color-word test, composed of three categories including color naming (color-congruent, eg, 'GREEN' in green), word naming, and interference (color-incongruent, eg, 'BLUE' in red), was conducted (Golden, 1978). The Grooved Pegboard Test (GPT) for motor performance of both dominant and nondominant hands was also conducted (Matthews and Klove, 1964).

\section{Statistical and Image Analysis}

Group differences involving continuous and categorical data were evaluated using independent $t$-test and Fisher's exact test, respectively. For regions that exhibited tissue volume differences, we conducted additional exploratory analyses to study associations between gray and white matter volumes and clinical and neuropsychological variables, using partial correlation and multiple linear regression analyses. Statistical significance was defined at an $\alpha<0.05$, two-tailed.

\section{RESULTS}

\section{Subjects}

Forty-one cocaine-dependent subjects and 41 sex-matched healthy comparison subjects were recruited for this study. One image from the patient group was excluded owing to the consistent problems with segmentation during the processing of the MR images for SPM analysis. Consquently, the analyses were conducted with 40 cocainedependent and 41 healthy comparison subjects. The mean age of cocaine-dependent subjects ( $41.4 \pm 6.9$ years old) was not significantly different from that of healthy comparison subjects $(38.7 \pm 8.8$ years old) ( $t$-test, $t(79)=1.57, p=0.12)$.
Table I Demographic and Clinical Characteristics of CocaineDependent and Healthy Comparison

\begin{tabular}{|c|c|c|c|c|}
\hline \multirow[b]{2}{*}{ Subjects } & \multicolumn{2}{|c|}{$\begin{array}{l}\text { Cocaine-dependent } \\
\text { subjects }(n=40)\end{array}$} & \multicolumn{2}{|c|}{$\begin{array}{c}\text { Comparison subjects } \\
(n=4 I)\end{array}$} \\
\hline & Mean & SD & Mean & SD \\
\hline Age $^{\mathrm{a}}$ (years) & 41.4 & 6.9 & 38.7 & 8.8 \\
\hline Education $^{\mathrm{b}}$ (years) & 12.5 & 1.83 & 14.3 & 1.74 \\
\hline $\begin{array}{l}\text { Nicotine use in past } \\
30 \text { days } s^{b}\end{array}$ & 16.8 & 11.4 & 0.87 & 4.17 \\
\hline Cocaine use (years) & 15.3 & 6.3 & - & - \\
\hline \multirow[t]{2}{*}{$\begin{array}{l}\text { Cocaine use in prior } \\
\text { month (days) }\end{array}$} & 17.8 & 8.4 & - & - \\
\hline & $n$ & $\%$ & $n$ & $\%$ \\
\hline $\operatorname{Sex}^{\mathrm{a}}$ (men) & 27 & 67.5 & 26 & 63.4 \\
\hline Handedness $^{\mathrm{a}}$ (right) & 38 & 95.0 & 37 & 90.2 \\
\hline $\begin{array}{l}\text { Alcohol abuse/ } \\
\text { dependence }{ }^{c}\end{array}$ & 20 & 52.6 & 0 & 0 \\
\hline Current & 12 & 31.5 & 0 & 0 \\
\hline Past & 8 & 21.1 & 0 & 0 \\
\hline
\end{tabular}

Abbreviations: - , not applicable; SD, standard deviation.

${ }^{a}$ There was no significant difference between the groups in age $(t(79)=1.57$, $p=0.12)$, sex composition $\left(\chi^{2}=0.15, p=0.70\right)$, or handedness $\left(\chi^{2}=0.67\right.$,

$p=0.4 \mathrm{I})$ between cocaine-dependent and comparison groups.

${ }^{\mathrm{b}}$ There was significant difference in educational level $(t=3.97, p<0.00 \mathrm{I})$ and nicotine use in past 30 days $(t=-6.4 \mathrm{I}, p<0.00 \mathrm{I})$ between groups.

'The number of subjects for alcohol abuse/dependence is 38 for the cocainedependent group.

There were no significant differences in sex composition ( $N=27,67.5 \%$ and $N=26,63.4 \%$, number of men in cocaine-dependent and healthy comparison groups, respectively) or handedness $(N=38,95.0 \%$ and $N=37,90.2 \%$, the number of right-handed subjects in cocaine-dependent and healthy comparison groups, respectively) between groups. Educational level was higher in the healthy comparison group than cocaine-dependent group $(t=3.97, p<0.001)$.

Details of demographic and clinical characteristics are presented in Table 1.

\section{Optimized VBM}

There were significantly lower gray matter volumes in bilateral premotor cortex (Brodmann area (BA) 6, 8, 16.6\%), right orbitofrontal cortex (BA 10,15.1\%), bilateral temporal cortex (BA 20,38, 15.9\%), left thalamus (12.6\%), and bilateral cerebellum $(13.4 \%)$ in the cocaine-dependent group relative to the comparison group (corrected $p<0.05$, height threshold $t=4.70$, and the extent threshold $=400$ voxels) (Figure 1, Table 2).

There was significantly lower white matter volume in right cerebellum (Talairach coordinates $x=25, y=-67$, $z=-41,552$ voxels; $10.0 \%$ ) in the cocaine-dependent group relative to the comparison group (corrected $p<0.05$, height threshold $t=4.17$, and the extent threshold $=400$ voxels). Both cerebellar gray and white matter volume differences were identified in the hemispheric, but not in the vermis (Figure 1). 

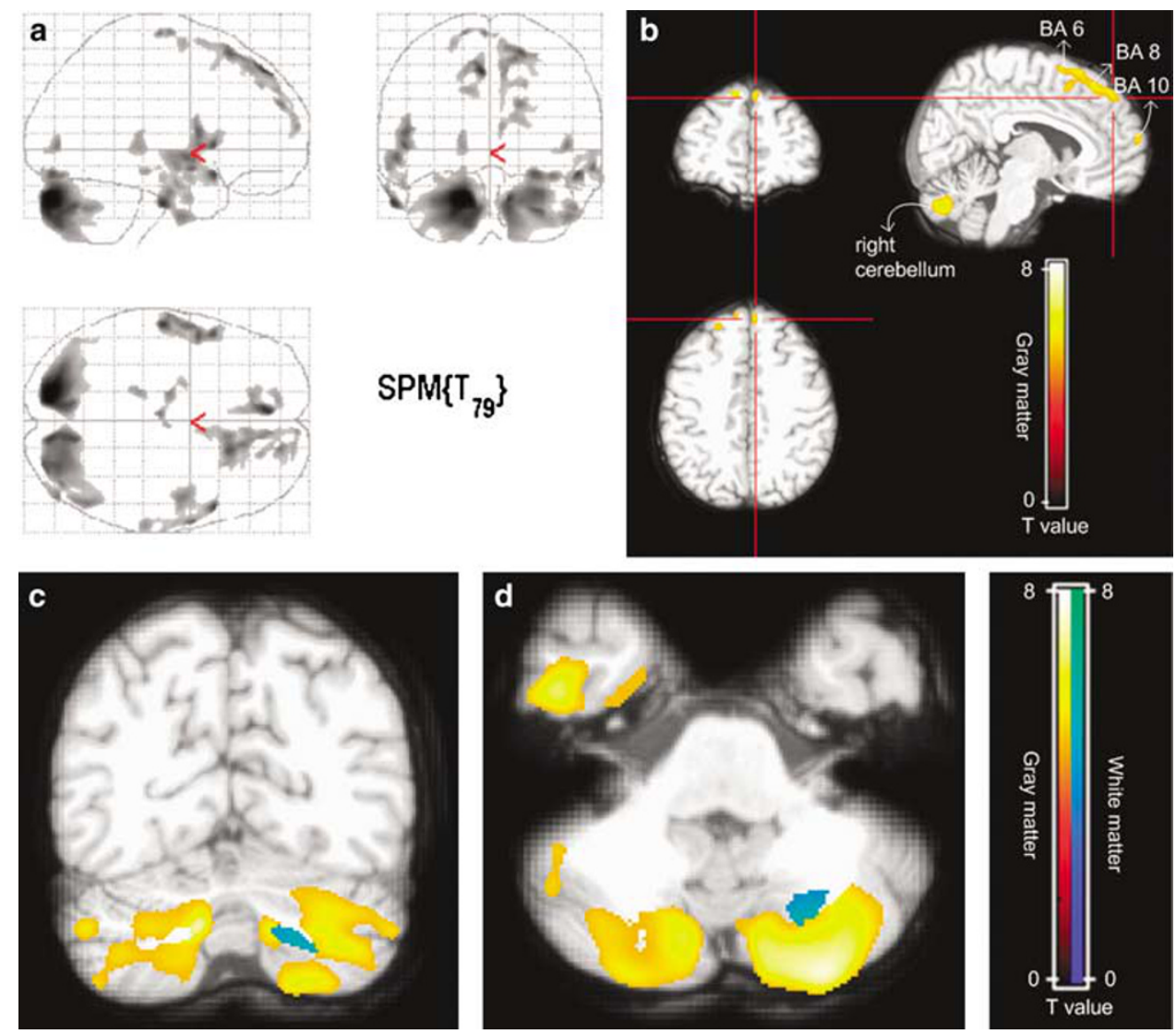

Figure I Location and degree of lower gray matter volume in cocaine-dependent subjects. (a) Locations of lower gray matter volume. (b) Frontal cortex gray matter volume differences. (c) Bilateral cerebellar gray and white matter volume differences (coronal view). (d) Bilateral cerebellar gray and white matter volume differences (axial view). Locations of lower gray matter volume in cocaine abusers are depicted in whole brain glass view (a). Gray matter volume differences between cocaine abusers $(n=40)$ and comparison subjects $(n=41)$. Cocaine abusers had lower gray matter volumes in bilateral premotor cortex (Brodmann area (BA) 6, 8, 16.6\%), right orbitofrontal cortex (BA 10, 15.1\%) (b), bilateral temporal cortex (BA 20, 38, I5.9\%), left thalamus (12.6\%), and bilateral cerebellum (13.4\%) as well as lower white matter volume in right cerebellum (10.0\%). Depicted color-defined cerebellar gray and white matter lesions are highlighted in yellow and blue, respectively (c and d). SPM map (gray matter: $p<0.05$ corrected for multiple comparisons, height threshold $t=4.70$, extent threshold $=400$ isotropic voxels of $1.0 \mathrm{~mm}^{3}$; white matter: $p<0.05$ corrected for multiple comparisons, height threshold $t=4.17$, extent threshold $=400$ isotropic voxels of $1.0 \mathrm{~mm}^{3}$ ).

\section{Correlation Analyses between Cerebellar Gray/White Matter Volume Changes and Clinical and Neuropsychological Variables within the Cocaine-Dependent Group}

Bilateral cerebellar gray matter volumes were negatively correlated with years of cocaine use $(r=-0.37, n=36$, $p=0.027 ; r=-0.39, n=36, p=0.018$; right and left, respectively) (Figure 2). Additionally, correlation analysis results between upper two variables with alcohol and nicotine-use history (the presence of comorbid lifetime and present alcohol abuse or dependence and nicotine use in past 30 days, respectively) as covariates have shown similar level of correlations $(r=-0.37, n=36, p=0.040 ; r=-0.37$, $n=36, p=0.036$; right and left, respectively). No other regions exhibited correlations between gray matter volume and any measure of cocaine use.

Partial correlation analysis results controlling for age, sex, and educational level in the cocaine-dependent group are as follows (Figure 3): left cerebellar gray matter volume was negatively correlated with the Trails B time $(r=-0.36$, $n=40, p=0.032)$. Bilateral cerebellar gray matter volumes were inversely associated with Stroop color-word test interference time $(r=-0.35, n=39, p=0.039 ; r=-0.47$, $n=39, p=0.005$, right and left cerebellum, respectively). Left cerebellar gray matter volume was related to GPT completion time for the nondominant hand $(r=-0.38$, $n=37, p=0.030$ ). Right cerebellar white matter volume also was negatively correlated with GPT completion time for both dominant and nondominant hands $(r=-0.37, n=37$, $p=0.036 ; r=-0.37, n=37, p=0.032$, respectively).

\section{Sex Differences}

The gray and white matter volume differences in male cocaine-dependent $v s$ male comparison groups ranged from 11.1 to $18.0 \%$ across target brain regions. Comparable gray and white matter volume differences ranging from 5.8 to $17.2 \%$ across target brain regions were found in female cocaine-dependent subjects relative to female comparison subjects. Multiple regression analyses indicated that there were no significant sex differences in gray or white matter volume differences. 
Table 2 Talairach Coordinates and Brodmann Areas for Regions of Lower Gray Matter Volume in Cocaine-Dependent Subjects ${ }^{\mathrm{a}}$

\begin{tabular}{|c|c|c|c|c|c|c|c|}
\hline \multirow{2}{*}{$\begin{array}{l}\text { Brain region } \\
\text { Right superior frontal cortex }\end{array}$} & \multirow{2}{*}{$\frac{\text { Brodmann area }}{6}$} & \multicolumn{3}{|c|}{ Talairach coordinates $[x, y, z]$} & \multirow{2}{*}{$\begin{array}{c}\text { Actual spatial extent } \\
2,646\end{array}$} & \multirow{2}{*}{$\frac{\text { z-score }}{5.64}$} & \multirow{2}{*}{$\frac{\boldsymbol{p} \text {-value }}{0.000}$} \\
\hline & & 18 & 24 & 59 & & & \\
\hline Left superior frontal cortex & 6,8 & -6 & 45 & 48 & 1,267 & 5.94 & 0.000 \\
\hline Right orbitofrontal cortex & 9,10 & 17 & 55 & 27 & 760 & 5.32 & 0.001 \\
\hline Right superior temporal cortex & 38 & 53 & 14 & -16 & ।,77। & 5.73 & 0.000 \\
\hline Right inferior temporal cortex & 20 & 47 & -4 & -41 & 1,090 & 5.28 & 0.000 \\
\hline Right cerebellum & & 12 & -85 & -30 & $|7,45|$ & 5.86 & 0.000 \\
\hline Left cerebellum & & -16 & -85 & -30 & $|9,03|$ & 6.43 & 0.000 \\
\hline
\end{tabular}

${ }^{a} p$-value $<0.05$ (corrected for multiple comparisons), height threshold $t=4.17$, cluster size $=400$ was defined as significant.
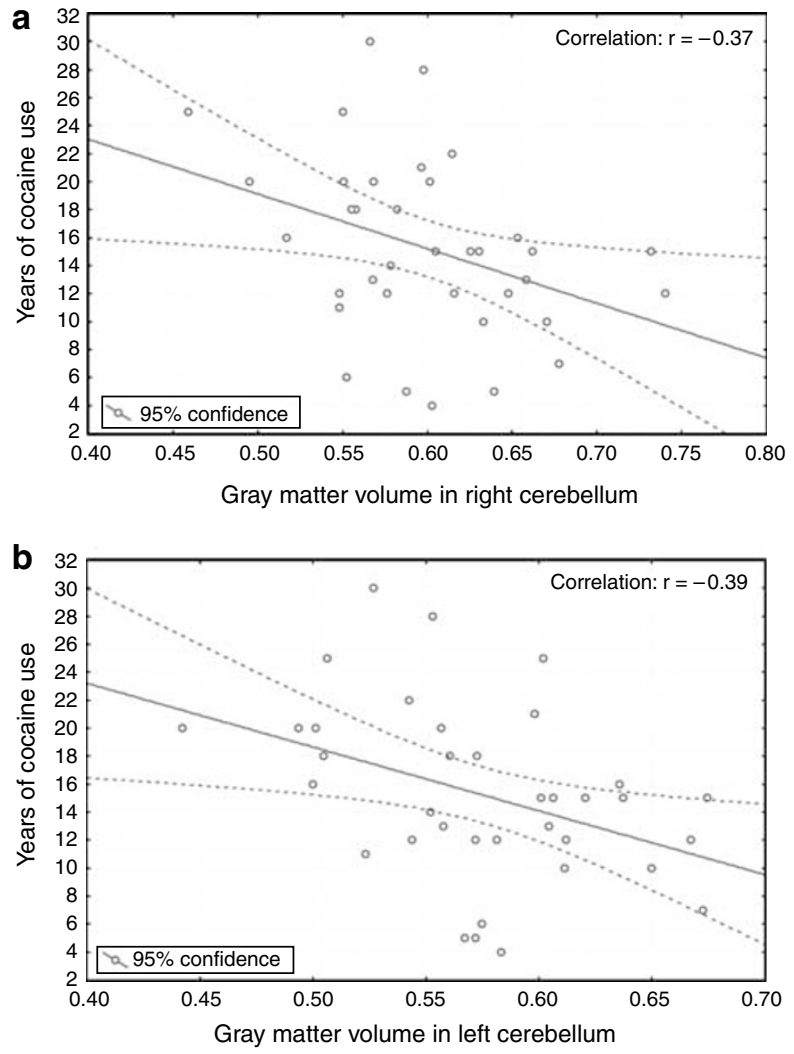

Figure 2 Correlations between cerebellar gray matter volumes and years of cocaine use. (a) Right cerebellum. (b) Left cerebellum. Right and left cerebellar gray matter volumes were negatively correlated with years of cocaine use $(r=-0.37, n=36, p=0.027 ; r=-0.39, n=36, p=0.018$, respectively) ( $a$ and $b)$.

\section{Aging Effects}

There were significant inverse correlations between age and gray matter volumes of bilateral premotor, temporal regions, and cerebellum in cocaine-dependent subjects ( $r$ : -0.38 to $-0.49, n=40, p<0.016)$. In healthy volunteers, this relationship was observed in bilateral premotor and cerebellar regions $(r:-0.35$ to $-0.43, n=41, p<0.027)$.

In all regions of significant difference, the diagnosis of cocaine dependence was a predictor for lower gray matter volume controlling for age. For bilateral cerebellum and premotor regions, age was a predictor for lower gray matter volume controlling for the diagnosis.

Considering this univariate relationship with age, an additional set of VBM analyses comparing cocaine-dependent and healthy comparison groups with age as a covariate was conducted. Similar results were produced to those when age was not covaried.

There were no correlations between age and cerebellar white matter volume in cocaine-dependent subjects.

\section{DISCUSSION}

\section{Cerebellar Abnormalities}

This study, the first optimized VBM analysis in cocainedependent subjects, is the first to document substantial gray and white matter cerebellar volume changes in cocaine abusers. Our finding that duration of cocaine use is correlated inversely with gray matter volume in cerebellum suggests that there may be some cumulative effect of cocaine on cerebellar gray matter tissue volume, such as has been observed in prefrontal areas (Liu et al, 1998).

The cerebellar abnormalities observed in cocaine abusers may result from a cerebellar sensitivity to oxidative stress (Pae et al, 2005), which can be a consequence of cocaineinduced cerebral vasoconstriction (Kaufman et al, 1998). As the cerebellum exhibits the highest nitric oxide synthase (NOS) activity in brain (Ikeda et al, 1993), oxidative stress, as caused by the cocaine-induced vasoconstriction, could promote increased formation of reactive nitrogen species (Rodrigo et al, 2001) and neuronal damage or death in cerebellum. The fact that we found a selective white matter volume difference only in cerebellum reinforces our hypothesis that the cerebellum may be an important target for cocaine's chronic effects.

However, it is also possible that these deficits might have been present before cocaine use. Consequently, further study that evaluates the difference in cerebellar gray matter volumes before and after cocaine use could clarify this issue.

In this study, lower bilateral cerebellar gray matter volumes were associated with deficits in executive function and motor performance in cocaine abusers. As the cerebellum has been implicated in executive function and motor control (Gottwald et al, 2004; Schmahmann and Sherman, 

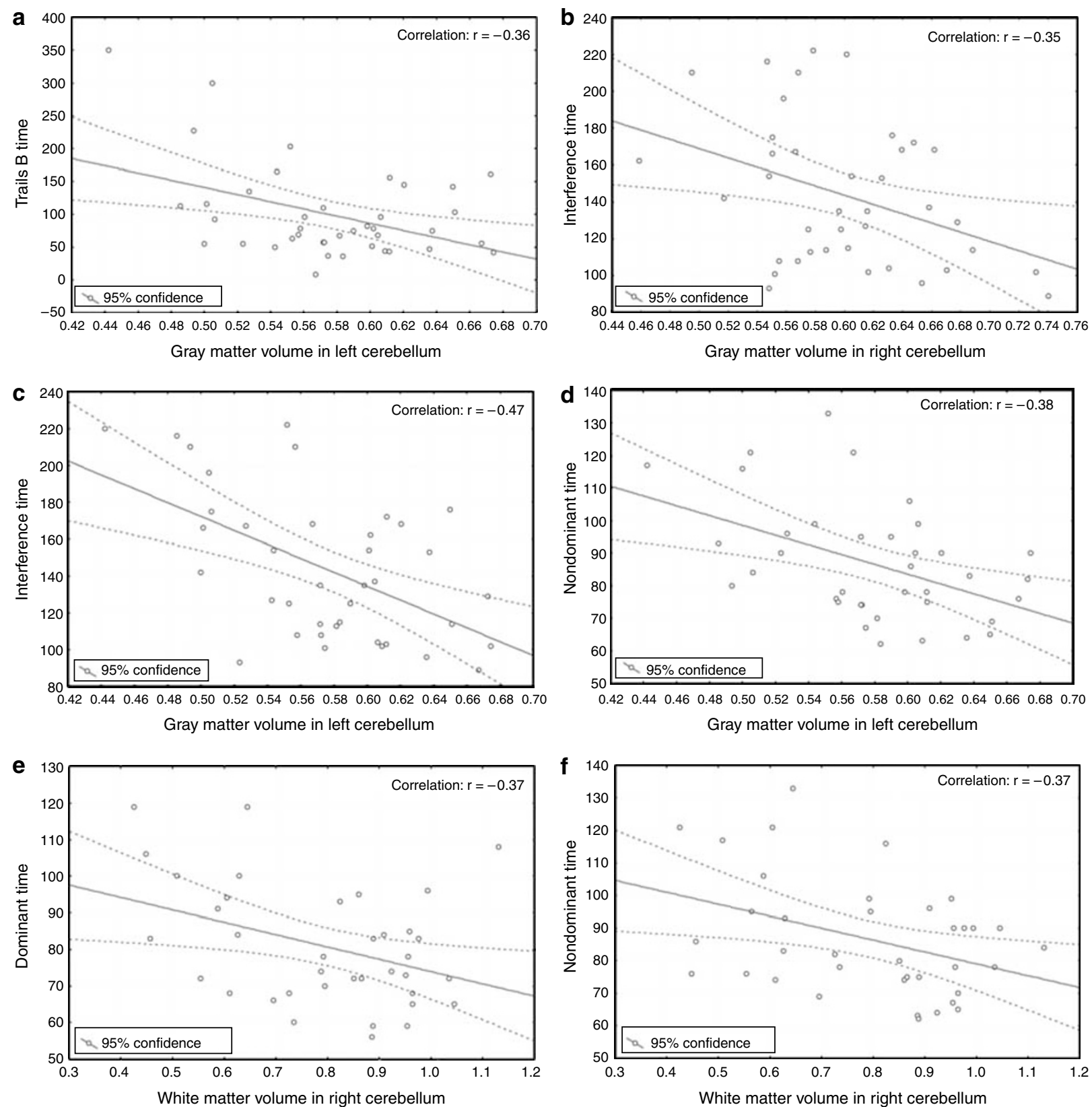

Figure 3 Correlations between cerebellar gray and white matter volumes, and neuropsychological function tests in cocaine-dependent subjects. (a) Left cerebellar gray matter volume and Trails B time. (b) Right cerebellar gray matter volume and Stroop color-word test interference time. (c) Left cerebellar gray matter volume and Stroop color-word test interference time. (d) Left cerebellar gray matter volume and GPT completion time with nondominant hand. (e) Right cerebellar white matter volume and GPT completion time with dominant hand. (f) Right cerebellar white matter volume and GPT completion time with nondominant hand. Left cerebellar gray matter volume was negatively correlated with the Trails B time $(r=-0.36, n=40, p=0.032)$ (a). Bilateral cerebellar gray matter volumes were inversely associated with the Stroop color-word test interference time $(r=-0.35, n=39, p=0.039 ; r=-0.47, n=39$, $p=0.005$, right and left, respectively) ( $b$ and $c$ ). Left cerebellar gray matter volume was related to GPT completion time for nondominant hand $(r=-0.38$, $n=37, p=0.030$ ) (d). Right cerebellar white matter volume was negatively correlated with GPT completion time for both dominant and nondominant hands $(r=-0.37, n=37, p=0.036 ; r=-0.37, n=37, p=0.032$, respectively) (e and $\mathrm{f}$ ). GPT, Groove pegboard test.

1998), these associations suggest that cognitive deficits in cocaine abusers may be, in part, due to this cerebellar deficit.

The cerebellar gray and white matter volume differences we observed were limited to bilateral cerebellar hemispheres, with no evidence of changes in cerebellar midline structures including the vermis. Interestingly, we have observed recently that cocaine-dependent subjects display an increased cerebellar vermis activation to cocaine- related stimuli as compared with healthy volunteers (Anderson et al, 2006). Because the cerebellar vermis has very high structural variability (Schmahmann et al, 1999), it is also possible that any potential vermis volume changes were undetectable with the present VBM method.

In the first VBM study of cocaine abusers, cerebellar abnormalities were not identified (Franklin et al, 2002). The discrepancy between the current and previous findings may 
arise from several factors including the statistical power and VBM technique. First, the statistical power for detecting significant differences was about 0.60 in Franklin et al (2002)'s report with a sample size of 13 cocaine-dependent subjects and 16 healthy volunteers, and an effect size of $8 \%$ mean difference. Brain regions exhibiting similar differences may not have been detected with this level of power. With our larger sample size $(n \geqslant 40$ each for cocainedependent subjects and comparison subjects), our statistical power exceeds 0.90 . Thus, based on sample size considerations alone, our study is likely to be more sensitive for detecting volume abnormalities. Second, we have adopted the optimized VBM method, whereas the standard VBM technique was utilized in an earlier study. There is evidence that the optimized VBM method is more sensitive than the standard VBM (Keller et al, 2004). Third, differences in demographic and clinical characteristics between these two cohorts may have contributed to the discrepancy.

\section{Cortical Abnormalities}

Franklin et al (2002) have reported decreased gray matter densities in ventromedial orbitofrontal, anterior cingulate, anteroventral insular, and superior temporal cortex. Subsequently, Matochik et al (2003), in a report focused on frontal lobe structures, documented decreased gray matter densities in bilateral anterior cingulate gyrus, medial orbitofrontal cortex, right lateral orbitofrontal cortex, and right middle/dorsal cingulate gyrus in cocaine abusers.

Constistent with our hypotheses as well as findings from previous studies (Franklin et al, 2002; Matochik et al, 2003), we found lower gray matter volumes in bilateral premotor cortex and right orbitofrontal cortex. These stuctural findings are also consistent with a prior functional neuroimaging study that demonstrated decreased prefrontal cerebral blood flow in cocaine-dependent subjects (Volkow et al, 1988).

The bilateral premotor cortex abnormalities we found were confined to rostral parts of the premotor cortex. As the rostral premotor cortex is associated with prefrontal cortex both structurally and functionally (Luppino et al, 1993; Hanakawa et al, 2002), gray matter deficits in our cocainedependent subjects may potentially be related to prefrontal functional deficits that have been reported in other cohorts (Volkow et al, 1988; Bolla et al, 2004).

Orbitofrontal abnormalities have been commonly reported in methamphetamine (Volkow et al, 2001) and cocaine-dependent subjects (Volkow et al, 1996). Deficits of this brain region has been associated with compulsive drug intake behaviors (Volkow and Fowler, 2000). There have been prior reports regarding the functional dysregulation of the orbitofrontal cortex in cocaine abusers (Volkow et al, 1991, 1993). We observed orbitofrontal gray matter deficits in cocaine-dependent subjects primarily on the right side. Lateralized changes in orbitofrontal cortices are constistent with prior reports that the right orbitofrontal cortex is more implicated in cocaine dependence (Bolla et al, 2003; Matochik et al, 2003).

As hypothesized, we also found lower gray matter volume in the bilateral temporal cortex, consistent with findings of a previous VBM study (Franklin et al, 2002). These findings are consistent with prior functional brain imaging studies which have reported decreased temporal cortex blood flow in cocaine abusers (Ernst et al, 2000; Gottschalk and Kosten, 2002).

There have been reports of frontal white-matter volume deficits in cocaine-dependent subjects (Schlaepfer et al, 2006). Schlaepfer et al (2006) have reported that substance abusers $(n=16)$ had smaller frontal white matter volume, but not in volumes of other brain regions, relative to healthy comparison subjects (Schlaepfer et al, 2006). In the current VBM study, however, there were no significant differences in frontal white matter volume between cocaine-dependent subjects and comparison subjects. This discrepancy may stem from differences in study methods (conventional region-of-interest method vs VBM).

\section{Subcortical Abnormalities}

We found lower left thalamic gray matter volume in the cocaine-dependent subjects. A prior magnetic resonance (MR) spectroscopy study reported decreased thalamic $\mathrm{N}$-acetyl aspartate levels, a marker for neuronal viability and density, in cocaine abusers ( $\mathrm{Li}$ et al, 1999). The thalamus serves as a relay between orbitofrontal cortex and nucleus accumbens and receives dopaminergic projections (Groenewegen et al, 1999). Therefore, it has been reported to play an important role in modulating reinforcing responses (George et al, 2001). Volkow et al (2003) have suggested that the thalamus may be involved in enhancement of the reinforcing effects of stimulants by expectation. Consequently, our finding of lower thalamic gray matter volume may be related to conscious drug-taking behaviors of cocaine-dependent subjects upon expectation of cocaineinduced euphoria.

\section{Comorbid Alcohol and Nicotine Abuse}

Alcohol abuse by cocaine-dependent cohorts can be an important confounding factor in research studies of human cocaine abusers, as alcohol consumption by itself induces neurochemical and neuroanatomical changes (O'Neill et al, 2001). It has been recently reported that alcohol has neurotoxic effects on cerbellum as measured by regionof-interest volumetry and VBM approach (Chanraud et al, 2007; Maschke et al, 2005; Sullivan et al, 2000). Accordingly, we instituted strict inclusionary/exclusionary criteria to minimize the effects of the alcohol confound in this study. In addition, correlation analyses between the duration of cocaine use and bilateral cerebellar gray matter volumes were conducted partialing out the alcohol history. The results remained unchanged relative to those without covariation. When the presence of comorbid lifetime alcohol dependence or abuse was covaried in the betweengroup VBM analysis, similar findings were also observed. On limiting our analyses to 20 cocaine-dependent subjects without lifetime history of alcohol dependence or abuse ( $v s$ all 41 comparison subjects), a similar pattern of gray matter volume deficits was observed.

Consequently, it may be suggested cautiously that effects of alcohol history on cerebellar gray matter volume may not be substantial in the current cohort.

Recently, Brody et al (2004) have reported that smokers have smaller gray matter densities in bilateral dorsolateral 
prefrontal cortex, left ventrolateral prefrontal cortex, and right medial cerebellum using standard VBM technique. Therefore, we conducted an additional VBM analysis with smoking as covariance to evaluate the potential confounding effect of smoking on our findings. However, similar regions of gray matter decrease in the cocaine-dependent subjects were observed. Consequently, we believe that our finding is not likely to be confounded by the presence of nicotine exposure.

\section{Effects of Aging}

Prior studies in cocaine-dependent subjects indicate a combined effect of aging and cocaine use on temporal lobe brain volume (Bartzokis et al, 2000). Consistent with that report, we found an age-related decline in temporal gray matter volume in cocaine-dependent subjects but not in healthy volunteers.

In addition, these age-associated gray matter volume declines in premotor regions were observed in both groups, a finding that also is consistent with the earlier report by Bartzokis et al (2000). There has been a report of ageassociated cerebellar atrophy in healthy volunteers (Raz et al, 2005). We also observed age-associated declines in cerebellar gray matter volumes in both cocaine-dependent and comparison groups.

The fact that cocaine-dependent subjects had lower gray matter volume relative to healthy volunteers after controlling for age suggests that cocaine and age may have additive effects for premotor and cerebellar gray matter differences.

Bartzokis et al (2002) have reported that an age-related increase in white matter volume of frontal and temporal regions were observed in healthy volunteers, but not in cocaine-dependent subjects and suggested that cocaineinduced vascular constriction may hamper the continued myelination of the adult brain. In this study, there were no associations between age and cerebellar white matter volume in either cocaine-dependent subjects or healthy comparison subjects. However, owing to the differences in analysis methods, the results cannot be directly compared.

\section{Limitations}

In this study, we did not control for the presence of certain psychiatric disorders including attention-deficit hyperactive disorder and antisocial personality disorder, which are highly prevalent in substance abusers (Johann et al, 2004).

Also, we cannot rule out the possibility that our findings may in part be due to a cohort effect. Caution should be used in interpreting our findings regarding the neuropsychological tests and partial correlation analyses, since multiple comparisons were conducted. We could also not exclude the potential effects of variable drug use history.

In conclusion, we found cerebellar gray and white matter volume differences as well as frontal, temporal, and thalamic gray matter volume differences. The cerebellum may be vulnerable to cocaine-associated structural changes, and cerebellar abnormalities may underlie some of neuropsychological deficits and motor dysfunction frequently observed in cocaine-dependent subjects. Owing to the cross-sectional nature of our study, further prospective studies are recommended to determine whether the cerebellar structural abnormalities we observed truly are cumulative with prolonged cocaine exposure as well as whether they are reversible with abstinence.

\section{ACKNOWLEDGEMENTS}

This work was supported, in part, by grants from the National Institute on Drug Abuse (DA09448-09S1, DA15116, DA50038, DA014674, and DA017324) (PFR, DAC, IKL, and MJK), Stanley Medical Research Institute and NARSAD (IKL and PFR), and Seoul National University Hospital (212004-008) (IKL), the National Institute on Alcohol Abuse and Alcoholism (K23AA13149, AA013727) (CCS and DAC), the VA Career Development Award (CCS), and the Brain Research Center of the 21st Century Frontier Research Program funded by the Ministry of Science and Technology, the Republic of Korea (70\% M103KV010022-06K220102210) (IKL).

\section{REFERENCES}

Anderson CM, Maas LC, Frederick BD, Bendor JT, Spencer TJ, Livni E et al (2006). Cerebellar vermis involvement in cocainerelated behaviors. Neuropsychopharmacology 6: 1318-1326.

Ashburner J, Friston K (1997). Multimodal image coregistration and partitioning - a unified framework. Neuroimage 6: 209-217.

Barroso-Moguel R, Mendez-Armenta M, Villeda-Hernandez J, Nava-Ruiz C, Santamaria A (2002). Brain lesions induced by chronic cocaine administration to rats. Prog Neuropsychopharmacol Biol Psychiatry 26: 59-63.

Bartzokis G, Beckson M, Lu PH, Edwards N, Rapoport R, Wiseman $\mathrm{E}$ et al (2000). Age-related brain volume reductions in amphetamine and cocaine addicts and normal controls: implications for addiction research. Psychiatry Res 98: 93-102.

Bartzokis G, Beckson M, Lu PH, Edwards N, Bridge P, Mintz J (2002). Brain maturation may be arrested in chronic cocaine addicts. Biol Psychiatry 51: 605-611.

Bolla K, Ernst M, Kiehl K, Mouratidis M, Eldreth D, Contoreggi C et al (2004). Prefrontal cortical dysfunction in abstinent cocaine abusers. J Neuropsychiatry Clin Neurosci 16: 456-464.

Bolla KI, Eldreth DA, London ED, Kiehl KA, Mouratidis M, Contoreggi $\mathrm{C}$ et al (2003). Orbitofrontal cortex dysfunction in abstinent cocaine abusers performing a decision-making task. Neuroimage 19: 1085-1094.

Brody AL, Mankelkern MA, Jarvk ME, Lee GS, Smith EC, Huang JC et al (2004). Differences between smokers and nonsmokers in regional gray matter volumes and densities. Biol Psychiatry 55: 77-84.

Chanraud S, Martelli C, Delain F, Kostogianni N, Douaud G, Aubin $\mathrm{HJ}$ et al (2007). Brain morphometry and cognitive performance in detoxified alcohol-dependents with preserved psychosocial functioning. Neuropsychopharmacology 32: 429-438.

Dager SR, Friedman SD, Parow A, Demopulos C, Stoll AL, Lyoo IK et al (2004). Brain metabolic alterations in medication-free patients with bipolar disorder. Arch Gen Psychiatry 61: 450-458.

Ernst T, Chang L, Oropilla G, Gustavson A, Speck O (2000). Cerebral perfusion abnormalities in abstinent cocaine abusers: a perfusion MRI and SPECT study. Psychiatry Res 99: 63-74.

First MB, Spitzer RL, Gibbon M, Williams JBW (1997). Structured Clinical Interview for DSM-IV Axis I Disorders, Research Version, Non-patient Edition (SCID-I/NP). New York State Psychiatric Institute, Biometrics Research: New York, NY.

Franklin TR, Acton PD, Maldjian JA, Gray JD, Croft JR, Dackis CA et al (2002). Decreased gray matter concentration in the 
insular, orbitofrontal, cingulate, and temporal cortices of cocaine patients. Biol Psychiatry 51: 134-142.

George MS, Anton RF, Bloomer C, Teneback C, Drobes DJ, Lorberbaum JP et al (2001). Activation of prefrontal cortex and anterior thalamus in alcoholic subjects on exposure to alcoholspecific cues. Arch Gen Psychiatry 58: 345-352.

Golden CJ (1978). Stroop Color and Word Test: A Manual for Clinical and Experimental Uses. Stoelting Company: Chicago, IL.

Good CD, Ashburner J, Frackowiak RS (2001a). Computational neuroanatomy: new perspectives for neuroradiology. Rev Neurol 157: 797-806.

Good CD, Johnsrude IS, Ashburner J, Henson RN, Friston KJ, Frackowiak RS (2001b). A voxel-based morphometric study of ageing in 465 normal adult human brains. Neuroimage 14: 21-36.

Gottschalk PC, Kosten TR (2002). Cerebral perfusion defects in combined cocaine and alcohol dependence. Drug Alcohol Depend 68: 95-104.

Gottwald B, Wilde B, Mihajlovic Z, Mehdorn HM (2004). Evidence for distinct cognitive deficits after focal cerebellar lesions. J Neurol Neurosurg Psychiatry 75: 1524-1531.

Groenewegen HJ, Galis-de Graaf Y, Smeets WJ (1999). Integration and segregation of limbic cortico-striatal loops at the thalamic level: an experimental tracing study in rats. J Chem Neuroanat 16: $167-185$.

Hanakawa T, Honda M, Sawamoto N, Okada T, Yonekura Y, Fukuyama $\mathrm{H}$ et al (2002). The role of rostral Brodmann area 6 in mental-operation tasks: an integrative neuroimaging approach. Cereb Cortex 12: 1157-1170.

Hester R, Garavan H (2004). Executive dysfunction in cocaine addiction: evidence for discordant frontal, cingulate, and cerebellar activity. J Neurosci 24: 11017-11022.

Ikeda M, Morita I, Murota S, Yuasa T, Miyatake T (1993). Distribution of nitric oxide synthase activity in rat brain-analysis by high performance liquid chromatography. Rinsho Shinkeigaku 33: 992-994.

Johann M, Bobbe G, Laufkotter R, Lange K, Wodarz N (2004). Attention-deficit hyperactivity disorder and alcohol dependence: a risk constellation. Psychiatr Prax 31: 102-104.

Kaufman MJ, Levin JM, Ross MH, Lange N, Rose SL, Kukes TJ et al (1998). Cocaine-induced cerebral vasoconstriction detected in humans with magnetic resonance angiography. JAMA 279: 376-380.

Keller SS, Wilke M, Wieshmann UC, Sluming VA, Roberts N (2004). Comparison of standard and optimized voxel-based morphometry for analysis of brain changes associated with temporal lobe epilepsy. Neuroimage 23: 860-868.

Kuhar MJ, Ritz MC, Boja JW (1991). The dopamine hypothesis of the reinforcing properties of cocaine. Trends Neurosci 14: 299-302.

Li SJ, Wang Y, Pankiewicz J, Stein EA (1999). Neurochemical adaptation to cocaine abuse: reduction of $\mathrm{N}$-acetyl aspartate in thalamus of human cocaine abusers. Biol Psychiatry 45: 1481-1487.

Lim KO, Choi SJ, Pomara N, Wolkin A, Rotrosen JP (2002). Reduced frontal white matter integrity in cocaine dependence: a controlled diffusion tensor imaging study. Biol Psychiatry 51: 890-895.

Liu X, Matochik JA, Cadet JL, London ED (1998). Smaller volume of prefrontal lobe in polysubstance abusers: a magnetic resonance imaging study. Neuropsychopharmacology 18: 243-252.

Luppino G, Matelli M, Camarda R, Rizzolatti G (1993). Corticocortical connections of area F3 (SMA-proper) and area F6 (preSMA) in the macaque monkey. J Comp Neurol 338: 114-140.

Lyoo IK, Streeter CC, Ahn KH, Lee HK, Pollack MH, Silveri MM et al (2004). White matter hyperintensities in subjects with cocaine and opiate dependence and healthy comparison subjects. Psychiatry Res 131: 135-145.

Maschke M, Weber J, Bonnet U, Dimitrova A, Bohrenkamper J, Sturm S et al (2005). Vermal atrophy of alcoholics correlate with serum thiamine levels but not with dentate iron concentrations as estimated by MRI. J Neurol 252: 704-711.
Matochik JA, London ED, Eldreth DA, Cadet JL, Bolla KI (2003). Frontal cortical tissue composition in abstinent cocaine abusers: a magnetic resonance imaging study. Neuroimage 19: 1095-1102.

Matthews CG, Klove H (1964). Instruction Manual for the Adult Neuropsychological Test Battery. University of Wisconsin Medical School: Madison, WI.

Miller NS, Millman RB, Keskinen S (1989). The diagnosis of alcohol, cocaine, and other drug dependence in an inpatient treatment population. J Subst Abuse Treat 6: 37-40.

O'Neill J, Cardenas VA, Meyerhoff DJ (2001). Separate and interactive effects of cocaine and alcohol dependence on brain structures and metabolites: quantitative MRI and proton MR spectroscopic imaging. Addict Biol 6: 347-361.

Pae EK, Chien P, Harper RM (2005). Intermittent hypoxia damages cerebellar cortex and deep nuclei. Neurosci Lett 375: 123-128.

Raz N, Lindenberger U, Rodrigue KM, Kennedy KM, Head D, Williamson A et al (2005). Regional brain changes in aging healthy adults: general trends, individual differences and modifiers. Cereb Cortex 15: 1676-1689.

Reitan RM (1992). Trail Making Test: Manual for Administration and Scoring. Reitan Neuropsychological Laboratory: Tucson, AZ.

Rodrigo J, Alonso D, Fernandez AP, Serrano J, Richart A, Lopez JC et al (2001). Neuronal and inducible nitric oxide synthase expression and protein nitration in rat cerebellum after oxygen and glucose deprivation. Brain Res 909: 20-45.

Schlaepfer TE, Lancaster E, Heidbreder R, Strain EC, Kosel M, Fisch $\mathrm{HU}$ et al (2006). Decreased frontal white-matter volume in chronic substance abuse. Int J Neuropsychopharmacol 9: 147-153.

Schmahmann JD, Doyon J, McDonald D, Holmes C, Lavoie K, Hurwitz AS et al (1999). Three-dimensional MRI atlas of the human cerebellum in proportional stereotaxic space. Neuroimage 10: 233-260.

Schmahmann JD, Sherman JC (1998). The cerebellar cognitive affective syndrome. Brain 121: 561-579.

Smelson DA, Roy A, Santana S, Engelhart C (1999). Neuropsychological deficits in withdrawn cocaine-dependent males. $\mathrm{Am} \mathrm{J}$ Drug Alcohol Abuse 25: 377-381.

Sullivan EV, Deshmukh A, Desmond JE, Mathalon DH, Rosenbloom MJ, Lim KO et al (2000). Contribution of alcohol abuse to cerebellar volume deficits in men with schizophrenia. Arch Gen Psychiatry 57: 894-902.

Volkow ND, Chang L, Wang GJ, Fowler JS, Ding YS, Sedler M et al (2001). Low level of brain dopamine D2 receptors in methamphetamine abusers: association with metabolism in the orbitofrontal cortex. Am J Psychiatry 158: 2015-2021.

Volkow ND, Ding YS, Fowler JS, Wang GJ (1996). Cocaine addiction: hypothesis derived from imaging studies with PET. J Addict Dis 15: 55-71.

Volkow ND, Fowler JS (2000). Addiction, a disease of compulsion and drive: involvement of orbitofrontal cortex. Cereb Cortex 10: 318-325.

Volkow ND, Fowler JS, Wang GJ, Hitzemann R, Logan J, Schlyer DJ et al (1993). Decreased dopamine D2 receptor availability associated with reduced frontal metabolism in cocaine abusers. Synapse 14: 169-177.

Volkow ND, Fowler JS, Wolf AP, Hitzemann R, Dewey S, Bendriem $B$ et al (1991). Changes in brain glucose metabolism in cocaine dependence and withdrawal. Am J Psychiatry 18: 621-626.

Volkow ND, Mullani N, Gould KL, Adler S, Krajewski K (1988). Cerebral blood flow in chronic cocaine users: a study with positron emission tomography. Br J Psychiatry 152: 641-648.

Volkow ND, Wang GJ, Ma Y, Fowler JS, Zhu W, Maynard L et al (2003). Expectation enhances the regional brain metabolic and the reinforcing effects of stimulants in cocaine abusers. J Neurosci 23: 11461-11468.

Worsley KJ, Marrett S, Neelin P, Vandal AC, Friston KJ, Evans AC (1996). A unified statistical approach for determining significant signals in images of cerebral activation. Hum Brain Mapp 4: 58-73. 